\title{
Una comprensión sobre el fenómeno del hecho religioso
}

\section{An Understanding of the Phenomenon of Religion}

Religiunmanta ruasqakuna, nisqakunapas yachanapaq

\section{Antonio Calderón ${ }^{1}$}

Universidad Católica del Maule, Talca, Chile

antocald@yahoo.es

\section{RESUMEN}

En términos generales, el presente estudio se aproxima a la comprensión del fenómeno y hecho religioso como una expresión social, histórica y cultural que determina el accionar social. De forma particular, intenta comprender cuáles son las características generales que definen esta práctica e inciden en la identidad colectiva e individual. También se analizan algunas características propias del hecho religioso y del pensamiento religioso: cómo y de dónde surge la idea de religión, en la cual la identidad individual y social se configura desde la religación. Para ello nos acercaremos a la sociología, la historia y la antropología, y luego veremos el aporte que Zubiri y la filosofía nos entregan para ir respondiendo a las interrogantes que surgen acerca del hecho religioso como un fenómeno social.

\section{PALABRAS CLAVE}

Filosofía, hecho religioso, religación, identidad, cultura

\section{ABSTRACT}

Our present study approaches the understanding of the phenomenon and religious fact as a social, historical and cultural expression that determines social action. In particular, understand what the general characteristics that define this social practice and how this affects religious cultural expression collective and individual identity. Some characteristics of religion and religious thought 
are also analyzed. How and where the idea of religion in which individual and social identity emerges, is configured from religation. To do this we will come to sociology, history, anthropology and then see the contribution that philosophy Zubiri and delivered us to go answering these questions that arise about the religious phenomenon as a social phenomenon.

\section{KEYWORDS}

Philosophy, religious fact, religation, identity, culture

\section{PISILLAPI QILLQASQA}

Kay qillqasqaqa religiunmanta yachanapaqmi. Tukuy ima nisqankuta, ruwasqakunamanta. Chaysi kanman ñoqanchikmanta yachay, ñaupa runakunamantapas yachana. Chayta yachaspa, runapa kasqanta riqsirisun. Maymantan hamura, imaynan paqarimura religiunmanta yachanaqa, kay pachakunapi. Chay tukuy imatapas yachanapaqmi Sociología nisqata, Historia nisqatapas, Antropología nisqatapas taqwirisun.

\section{TIQSI RIMANAKUNA}

Filosofía, harawikuna, religiun kasqanmanta, yachay, riqsiy

\section{Introducción}

Nuestra tarea a realizar tiene que ver con un análisis acerca de los procesos y las condiciones de las identidades colectivas e individuales presentes en todo ser humano a partir del hecho religioso. Para ello, acudimos a unas configuraciones propias que son componentes esenciales de toda sociedad humana. Nos referimos a la cultura y a la religión. Aunque estas dos dimensiones aparentemente parecen estar alejadas la una de la otra, encontramos que existen ciertas interrelaciones que se pueden presentar como elementos de suma importancia en la forma en que la dinámica social se desenvuelve en nuestra contemporaneidad. Nuestro propósito tiene relación con el aporte que hace Zubiri desde la filosofía al hecho religioso. Para ello seguiremos los siguientes pasos: 1) describir la comprensión del hecho religioso desde la sociología, la antropología y la historia; 2) enfocarnos desde el planteamiento y aporte que desde la filosofía realiza Zubiri al tema que nos convoca. 


\section{Comprensión sobre el hecho religioso a partir de algunas disciplinas humanistas}

La consideración y comprensión acerca del hecho religioso ha sido siempre un tema de alta complejidad, al involucrar un aspecto de lo humano al cual tributan y buscan responder otras disciplinas como la filosofía, la sociología y la historia. Así, surgen a menudo discusiones y desencuentros, pues cada una de ellas tiene una mirada del hombre propia. Sin embargo, se hace necesaria esta búsqueda multidisciplinaria, que busca dar una posible respuesta a dicho fenómeno que revela características comprensivas e incomprensivas a través del tiempo y de cada contexto, acerca del comportamiento de la propia sociedad y de los individuos.

Por un lado, está la perspectiva de Durkheim, quien presenta una visión muy crítica acerca de las ideas que presentan los evolucionistas en relación con la religión. Su teoría es la que tiene mayor influencia y relevancia en el pensamiento social contemporáneo, principalmente en la sociología y la antropología. Durkheim intentó superar las barreras en la definición sobre la religión y el hecho religioso, que se centraban solo en las creencias en dioses y espíritus. Es así que argumenta que la religión es un cuerpo de prácticas y creencias relativas a las cosas sagradas de manera absolutas; esto es, todo aquello que se identifica con los hechos dejados y prohibidos - creencias y prácticas - que dan solvencia y unidad a una comunidad moral concreta.

En el análisis que realiza Durkheim sobre los aspectos esenciales de las religiones se encuentra con que estas son antifuncionales, puesto que, como la ciencia ha demostrado, las religiones poseen equivocaciones con respecto a las características que posee la naturaleza y el universo (Durkheim, 1992, pp. 388-389). Aquí manifiesta y argumenta que es necesario el encuentro de las funciones que contiene la religión y el hecho religioso en términos más reales y mundanos; es decir, es aquello que le sucede a la sociedad que las lleva a cabo en tanto que la religión existe por la manera en que sirve de unión entre los seres humanos y asegura así la existencia social. Asimismo, Durkheim indica que las distintas religiones no se pueden considerar como falsas unas con otras, ya que existe una heterogeneidad de elementos frente a las múltiples necesidades fundamentales humanas que solamente las religiones y el hecho religioso de estas pueden solventar en el tiempo. Por lo tanto, no existen religiones ni hechos religiosos más especiales que otros.

En el texto se exponen algunos elementos de la corriente teórica de este pensador clásico, que a través de sus estudios logra precisar y generar bases sobre las que gira la comprensión del fenómeno y del hecho 
religioso. Su pensamiento logra influenciar las ciencias sociales y, por ese motivo, podemos hacer una distinción entre las características de lo que es el hecho religioso sin importar la caracterización de la tradición, debido a que los elementos constitutivos de la religión no son uniformes, ni en la forma en que se presentan ni en cómo son conceptualizadas.

Por otro lado, la antropología cultural tiene la misión de estudiar las características propias de la humanidad y del ser humano: sus creaciones, sus idiomas y sus formas de comunicación, como el lenguaje, sus modos de comportamiento, sus normas y leyes, la organización societal, la cultura y la religión. Con esta definición, nos acercamos a considerar a la antropología cultural como una disciplina dentro de las ciencias sociales, lo que nos permite conocer de manera consistente aspectos más formales de la religión y el hecho religioso. Es decir, se consideran como elementos viables de exteriorización y de interpretación de su realidad, más íntima y propia. Es necesario precisar que esta es una creación y recreación humana que surge de la sociedad misma; de ahí que los estudios que vayan surgiendo desde la visión antropológica son características propias del pensamiento religioso, así como la forma en que este se combina con las significaciones, símbolos y configuraciones propias de cada una de las sociedades (Bianchi y otros, 2001, p. 11).

Desde la perspectiva de la historia de las religiones, Eliade aborda el hecho religioso desde el comportamiento que poseen muchos grupos, desde la experiencia grupal y personal de lo divino por medio de la hierofanía, que consiste en la manifestación de un ser divino ante una persona o un grupo humano, de tal manera que se genera en ellos una experiencia vivencial y se logre así un momento de un hecho religioso, donde el recuerdo de la comunidad llega a ser tan peculiar y profundo que lo constituye en algo único (Eliade,1981, pp. 34-35). Precisamente este hecho experiencial se traduce como una forma de vida religiosa, debido a que el momento de lo sacro se manifiesta como un ser sagrado llamado Dios. Eliade considera que la historia de las religiones contiene muchas y variadas manifestaciones de consistencia hierofánicas, donde la vida sagrada y la profana son elementos claves para configurar lo social. El hecho religioso abarca desde la unión de lo sagrado con lo profano; aparece así un dualismo en el hecho religioso.

A partir de ahí el creyente considera dos diferencias esenciales: por un lado, está lo profano y, por otro lado, lo sagrado. El primero tiene que ver con las actividades cotidianas en que el ser humano se desenvuelve y desarrolla sus acciones. El segundo tiene que ver con el lugar preciso en 
que el ser humano logra contactarse con el ser divino ${ }^{2}$ por medio de un determinado proceso de purificación y obediencia, que conduce a una complementación de estos dos mundos por medio de los ritos (Eliade, 1981, p. 121).

Ahora bien, este hecho religioso de sentido hierofánico no desaparece en el tiempo, pese a que nos encontramos en una era de globalización. Por el contrario, se traduce en nuevas estructuras o formas no consideradas con parámetros muy distintos de los que antiguamente estudiaban los hechos o fenómenos religiosos. Esto obedece al desarrollo nuevo que alcanza la utilización de los medios de comunicación para poder transmitir nuevos elementos de información a través de distintas redes on-line a nivel mundial; ello lleva a una reestructuración nueva, donde las expresiones de lo hierofánico no desaparecen fácilmente.

\section{Algunas características propias del hecho religioso}

Antiguamente, el ser humano realizaba la comprensión de su medio a partir de lo natural. Por ende, no existía explicación alguna cuando realizaba la adoración a objetos y fenómenos naturales, y al mismo tiempo sentía una devoción por ellos en su forma material. Esta explicación se puede entender en el culto a diferentes hechos religiosos que realizaba el ser humano, que posteriormente se traducían en hechos sobrenaturales a los cuales les asignaba una serie de elementos simbólicos, generando así una manifestación divina.

Para Zambrano, la mirada que se tiene de los dioses es una de las características propias de la constitución humana, según la cual los seres humanos dotan a sus dioses de una manera absoluta ciertos atributos propios del hombre. Ello conduce a una situación simbólica del mismo hombre, de donde surge la idea de que Dios es perfecto e ilimitado, mientras que los seres humanos somos falibles y limitados (Zambrano, 2005, pp. 238-239).

Ahora bien, otro elemento importante es la comprensión de este hecho religioso en las acciones propias del ser humano cuando se enfrenta a una estructura de restricciones que se imponen en la vida cotidiana y profana por medio del tabú. Esta es una de las formas más claras y evidentes de la implicación de las configuraciones religiosas en la vida normal y cotidiana de los seres humanos, en la que aparece nuevamente una dupla que trata de lo prohibido y permitido, en la cual un grupo determinado indica aquello que es bueno y malo como la dimensión propia de la moral.

2 Zambrano (1995, p. 236) indica que los dioses son las formas de esas manifestaciones en el arcano, que se convierte en misterio. Y un misterio es algo ya accesible; es la forma en que un secreto, sin perder su condición, se manifiesta. 
Otra característica propia del hecho religioso tiene que ver con la fe, que logra explicitar mejor y de manera más clara este fenómeno. Ahora bien, al parecer muchos estudiosos de sociología, antropología e historia religiosa siguen sus estudios del hecho religioso solamente como una dimensión social, cultural e histórica, y no consideren particular atención en este punto, puesto que no logran concebir los elementos propios sobrenaturales contenidos en las configuraciones de los espíritus o del alma. Por medio de la fe como acto, el hecho religioso se sustenta y en él se sustentan los estamentos religiosos; de este modo, constituye el núcleo mismo de las expresiones en el diario vivir. Esto se fortalece por medio de los textos revelados o sagrados, y así conforman una característica propia de identidad como sociedad y su cultura al mismo tiempo (Bianchi y otros, 2001, p. 404). Esto genera una identidad societal, con las tradiciones escritas, los textos sagrados y las tradiciones orales, que, transmitidas a todos los miembros de un grupo humano, generan su propia particularidad dentro del contexto existente de los grupos religiosos en el mundo.

\section{La identidad de una sociedad es permeada por el hecho religioso}

Zambrano indica que varias sociedades antiguas humanas han logrado construir su identidad sobre la base del hecho religioso y de varias otras características como proceso. Para ello, es necesario realizar una búsqueda y comprender su influjo en los aspectos de la vida e identidad de una sociedad (Zambrano, 2005, p. 238). A fin de comprender esta construcción, consideramos esta pregunta: ¿a qué se debe que el ser humano le da mucha importancia a lo sagrado, considerando que lo profano es parte constante de su vida cotidiana?

Partimos respondiendo esta pregunta. Las consecuencias de las configuraciones sociales van construyéndose a partir de las interacciones humanas y religiosas, lo cual nos conduce a un estudio acerca de las religiones y el hecho religioso. Por un lado, se entiende que la religión y los hechos religiosos tienen como objetivo directo fortalecer la unión social. Por ejemplo, algunos grupos sociales aún consideran los fenómenos cósmicos como elemento indispensable y, por ello, invocan a los dioses de las Iluvias; además, por medio de bailes no solo buscan conscientemente la unión social, sino fortalecer su unificación, y esto de manera indirecta busca una cohesión social. Para Durkheim, así se busca la cohesión social bajo un pretexto: las invocaciones de las precipitaciones pluviales, pero también eso es parte de lo sagrado (Durkheim, 1992, p. 39). 
Aunque para muchos no es relevante, la religión es parte del ser humano. Incluso en su configuración social es un elemento fundamental para la composición del individuo y de su propia identidad, pese a las formas en cómo esta se presente y se organice. Esta configuración le imprime un sello y un carácter, y forma una unión social y un proceso histórico humano. Su importancia es tan fundamental que la religión es referencia para la sociedad; de ella surgen en gran parte las acciones morales y éticas. Por otro lado, también la religión determina la forma de pensar de las sociedades, lo que se ve plasmado en el individuo.

\section{Zubiri y el aporte desde la filosofía acerca del hecho religioso}

Desde la filosofía contemporánea, Zubiri nos plantea una sugerente proposición en relación con el fenómeno o hecho religioso: «se trata de que nos preguntemos en qué consiste formal y constitutivamente lo religioso de un hecho en cuanto tal» (Zubiri, 1994, p. 15). El propio Zubiri indica que existen dos vías: una es que las religiones son unos hechos que existen en las culturas humanas, y con razón Zambrano dice: «Una cultura depende de la calidad de sus dioses» (Zambrano, 2005, p. 27). Es decir, existen instituciones religiosas que poseen ritos, creencias y su puesta en práctica de ellas. La otra vía tiene que ver con la religión, cuyo objeto consiste en dedicarse en lo formal a lo sagrado.

Pero para comprender mejor este asunto, veamos los argumentos con que Zubiri sostiene sus postulados. Lo primero que debemos considerar es que la característica propia del realismo clásico es considerar a la realidad bajo el prisma del conocimiento del saber sobre lo real. En cambio, para el filósofo español la realidad tiene que ver con un modo, porque conocer y aprender la realidad no son dos actos distintos, puesto que la realidad se nos proporciona en el mismo saber, lo cual constituye un mismo acto. Así, el ser y el saber tienen una correspondencia mutua. Por tanto, para el ser humano la realidad no es otra cosa que el modo como se le presentan los elementos en el acto mismo de aprehenderla. De ello se comprende que la realidad es un modo en que los elementos o las cosas se nos dan en nuestra aprehensión. Aquí se da una unidad estructural de sentir e inteligir, por lo que el filósofo vasco indica que esa unidad estructural es inteligencia sentiente. Por lo mismo, las cosas son reales en cuanto inteligidas y aprehendidas como siendo de suyo, siendo en propio y siendo otras. La realidad es entonces formalidad debido a que las cosas se presentan y manifiestan en nuestra intelección sentiente con alteridad. Así entendida, «la realidad es cómo el hombre desde las cosas va a configurar el ser de su propia realidad sustantiva. El hombre, por consiguiente vive desde la realidad de lo real» (Zubiri, 1994, p. 38). Debido a esto, la realidad es inespecífica, abierta, transcendental. Por lo tanto, la realidad 
constatada es mucho más que cada una de las cosas y mucho más que nosotros mismos. Por ser demasiado, la realidad se impone y ejerce un poder sutil e inevitable sobre nuestras vidas. La religación es justamente este hecho radicalísimo y total: estamos atados a este poder. Es lo que Zubiri llama el poder de la realidad. La realidad es muy potente, poderosa y despliega su primacía en cada acto de nuestra existencia. Lo despliega como apoyo último, como fuente absoluta de posibilidades y como lanzamiento radical que nos impele a realizarnos de alguna manera. Este elemento fundamental lleva a Zubiri a indicar que los seres humanos somos absolutos, pero de manera relativa, puesto que somos parte de esta realidad como seres abiertos a ella y estamos frente a ella en la totalidad. Ahora bien, el problema esencial del ser humano tiene que ver entonces con la forma en que plasmará su vida en esta realidad. Cuando tiene enfrente una realidad de ultimidad, de posibilitación y de imposición elementos que caracterizan la realidad del ser sustantivo, aparece la radicalidad. Esa actitud radical es entendida por Zubiri como religación. "La religación es la ligadura a la realidad en cuanto realidad para ser» (Zubiri, 1994, p. 40). También dice: «la religación es ante todo un hecho constatable [...] es algo que afecta al todo de mi realidad humana [...] está religado al poder de lo real» (Zubiri, 2003, p. 93). No es una unión física ni una presión social, sino un enfrentarse con el carácter de realidad de todo ello.

Cuando el filósofo vasco introduce el término religación, no lo hace con la finalidad de explicitar el fenómeno religioso o los hechos religiosos, sino que explica que es un elemento con una dimensión elemental y constitutiva propia de la existencia humana, en el que todos los seres humanos estamos afectados. Por ende, viabiliza la existencia de diversas religiones $y$, además, se entiende que otras personas simplemente indican su parecer y deciden de modo explícito no concebir ninguna religión.

Cuando sugiere explicitar el tema de la religación, lo hace pensando en que ella genera una unión ontológica a lo que nos hace ser. Por lo tanto, la religación se presenta como una nueva manera de lograr una validación sobre el problema de Dios en el contexto del pensamiento contemporáneo. Así versa en todo su planteamiento acerca de Dios, que se transforma en un fundamento explicativo de todo el problema. Ahora el problema de Dios se entiende y comprende de manera muy clara, debido a que ya se encuentra planteado en la religación esencial de la existencia humana, que no es nada más que la dimensión previa a toda demostración racional de la existencia de Dios.

De esta forma, la religación se actualiza de modo formal, pero unificado al universo material que se muestra por la claridad ejercida en lo fundamental de religado. El ser humano opta por considerar una religión 
positiva o no; sin embargo, esto siempre consistirá en la religación, que es su dimensión radical y personal. Pero esta religación no explica la realidad certera de un Dios especifico, sino que se coloca dentro del ámbito de la deidad y se presenta como el elemento fundante. «Deidad no es Dios. Le llamo deidad por dos razones; porque será justamente la vía que nos conduzca a Dios, y además porque en última instancia el hombre ha sentido siempre como un poder de deidad ese carácter universal y dominante que la realidad en cuanto tal tiene sobre él y sobre todas las cosas que son reales. La deidad no es nada distinto del mundo y de las cosas reales» (Zubiri, 1994, pp. 43-44). De ahí que el primer atributo que descubrimos de la divinidad es su fundamento. Por tanto, la religión es considerada como una plasmación posible de la religación. Sin embargo, también el ateísmo y el agnosticismo son posibles plasmaciones de la misma religación. Para Zubiri, la afirmación de que el teísta, el ateo y el agnóstico aquí están considerados en igualdad de condiciones implica que no existen ciertos privilegios de uno frente a los otros. En suma, las religiones son conjuntos sociales radicalmente históricos y con consideraciones de mucha variabilidad.

\section{La comprensión del hecho religioso desde lo fundante y el hecho de la religalidad}

El análisis del hecho radical de la religación, que Zubiri plantea como fundamental, es para una mejor comprensión y explicación de lo que es religión. A partir de ello, esperemos que el filósofo pueda otorgarnos una definición universal de religión. Pero debe ser una definición en la que no exista una imposición de lo concebido como religión frente a otras tradiciones religiosas. La forma de proceder en Zubiri es considerar una cierta neutralidad interpretativa, porque el estudio de la religación no necesita una atención especial y sensibilidad religiosa. Por el contrario, es un método que posibilita una ventaja comparativa de aceptar la especificidad de lo religioso, precisamente debido a la no reducción de solo procesos sociológicos, históricos o antropológicos. A pesar de todo, no se puede dejar de lado la fenomenología interpretativa, puesto que realiza un claro análisis de los hechos religiosos, aunque muy posterior al análisis del hecho de la religación.

Ahora bien, Zubiri no se queda con los supuestos que trata la sociología con el método de la fenomenología-hermenéutica, ni argumenta en contra acerca de la explicación histórica sobre la religión. Nos invita a que observemos de forma muy peculiar una fenomenología de la religión, considerando la estructura primaria y radical de la religación como partida del hecho primordial de la religación o los hechos religiosos, como poder de lo real. 


\section{La estructura de la religación como poder de lo real}

Por lo visto hasta aquí, queda claro que el poder de lo real fundamenta al ser humano con un empoderamiento. Este empoderamiento permite que la realidad sea de modo intrínseco y formalmente constitutivo de esta. Sin este empoderamiento, el ser humano no actuaría como tal. El ser humano no es nada sin las cosas y la realidad. Zubiri indica que el ser humano «Necesita que le permitan hacerse, porque si bien tiene poder $y$ deber de hacerse, precisa de un impulso para hacerse, el cual es su capacidad de poder que tiene de lo real» (Zubiri, 2003, pp. 92-94).

Por lo tanto, no es que la realidad ayude al hombre a vivir, sino que ella le permite al hombre ser real. Solamente a partir del estar pendiente del poder de lo real es que el ser humano se comprende como realidad (Solari, 2010, p. 181). La religación entonces tiene una solvencia ligante a la realidad en cuanto realidad para ser. Así se demuestra que este acto es constatable, básico y radical. Por tanto, como la realidad es fundamento último, posibilitante e impelente al ser humano, así también el poder de lo real nos condiciona físicamente a ser absolutos, religándonos a él como último, posibilitante e impelente (Solari, 2010, p. 181). Por ello, la religación al poder de lo real no es una unión material que sujeta la persona, «sino que está constitutiva y formalmente religada al poder de lo real» (Zubiri, 2003, p. 93). Finalmente, la religación llega a ser experiencial, no conceptivo sino físico. Para Zubiri, esto no constituye ser teoría verificable o una interpretación, sino que, por el contrario, es un acto constatable con el que se encuentra todo ser humano, pese a que lo conozca o no. La religación es un hecho básico y radical de toda posible vida humana que se puede descubrir de muchas maneras, pero no se puede demostrar, lo que se da en la aprehensión de religación.

\section{Conclusión}

Llegando al final de este trabajo y considerando nuestra contemporaneidad, los problemas fundamentales acerca del fenómeno y la comprensión sobre el hecho religioso - los cuales son considerados por distintas disciplinas humanistas anteriormente- nos indican que en cada tiempo y contexto necesitamos analizar y considerar un replanteo sobre ellas. Hoy se hace urgente explicitarlo mejor y de manera muy simple a los seres humanos.

A partir de ello, Zubiri nos lleva a explorar precisamente acerca del poder de lo real entendido desde la religación: a partir de ahí el ser humano comprende su actitud radical ante la realidad de las cosas, por su trasfondo de realidad que tiene que ver con la realidad y su estructura trascendente. Si comprendemos este primer aspecto del hecho religioso 
bajo el prisma de la religación, entonces el ser humano, en la plenitud de su estar y su ser, se concebirá religado a Dios. De ello se desprende que el ser humano en la plenitud de su ser se encuentra religado a Dios como su máxima posibilidad, que lo hace ser él mismo. Por tanto, podemos comprender que el hecho religioso y el poder de lo real en el religamiento que Zubiri plantea nos permiten visualizar prácticamente muchos elementos desde su propia filosofía. 


\section{REFERENCIAS BIBLIOGRÁFICAS}

Bianchi, U. y otros. (2001). Tratado de antropología de lo sagrado. Madrid: Trotta Editorial.

Durkheim, E. (1992). Las formas elementales de la vida religiosa. Madrid: Akal Ediciones.

Eliade, M. (1981). Tratado de historia de las religiones. Madrid: Cristiandad Ediciones.

Solari, E. (2010). La raíz de lo sagrado. Santiago de Chile: Ril Editores.

Zambrano, M. (2005). El hombre y lo divino. México D. F.: Fondo de Cultura Económica.

Zubiri, X. (2003). El hombre y Dios. Madrid: Alianza Editorial. (1994). El problema filosófico de la historia de las religiones. Madrid: Alianza Editorial. 